\title{
Trombosis venosa portal extrahepática, manejo quirúrgico con derivación meso-Rex. Serie de 3 casos
}

\author{
Extrahepatic portal vein thrombosis, surgical management with \\ meso-Rex shunt. Series of 3 cases
}

\author{
Cesar Eduardo Jiménez ${ }^{1,2} \mathbb{D}$, Leonardo Randial ${ }^{1} \mathbb{D}$, Iván Silva $\mathbb{D}^{\mathbb{D}}$, \\ Manuel Hossman-Galindo ${ }^{1}$ DD, Mario Bravo ${ }^{3}$, Osiris Moreno ${ }^{4} \mathbb{D}$
}

\begin{abstract}
Médico, especialista en Cirugía vascular, Hospital Universitario clínica de San Rafael, Bogotá, D.C., Colombia. Jefe, Servicio de cirugía vascular, Hospital Universitario clínica de San Rafael, Bogotá, D.C. Colombia.

Médico hospitalario, Servicio de cirugía vascular, Hospital Universitario clínica de San Rafael. Bogotá, D.C., Colombia.

Médico, residente de Cirugía general, Universidad del Bosque. Bogotá, D.C., Colombia.
\end{abstract}

\section{Resumen}

La vena porta es un conducto que drena el flujo esplácnico al hígado y se puede ocluir por diferentes patologías, variando su presentación clínica de acuerdo con la causa de la obstrucción. Es muy importante diferenciar la trombosis portal asociada o no a la cirrosis, ya que su tratamiento y pronóstico es diferente. La trombosis venosa portal extrahepática es una condición netamente de origen vascular, y es la principal causa de trombosis portal en niños y adultos. Presentamos tres casos tratados con derivación meso-Rex, con seguimiento a 6 meses.

Palabras clave: meso-Rex; vena porta; trombosis venosa; derivación portocava quirúrgica; várices.

\begin{abstract}
The portal vein is a conduit that drains splanchnic flow to the liver, it can be occluded by different pathologies and its clinical presentation varies according to the cause of the obstruction. It is very important to differentiate portal thrombosis associated or not with cirrhosis, since its treatment and prognosis is different. Extrahepatic portal vein thrombosis (PEVT) is a condition of purely vascular origin, being the main cause of portal thrombosis in children and adults. We present three cases with meso-Rex shunt, with a 6-month follow-up.
\end{abstract}

Keywords: meso-Rex; portal; venous thrombosis; surgical portacaval shunt; varicose veins.

Fecha de recibido: 05/03/2020 - Fecha de aceptación: 15/06/2020

Correspondencia: Cesar Eduardo Jiménez, Calle 17 No 16-68, Casa 18, Chía, Colombia. Teléfono: 3142976816

Correo electrónico: cesarejmd@yahoo.com

Citar como: Jiménez CE, Randial L, Silva I, Hossman-Galindo M, Bravo M, Moreno O. Trombosis venosa portal extrahepática, manejo quirúrgico con derivación meso-Rex. Serie de 3 casos. Rev Colomb Cir. 2021;36:98-109. https://doi.org/10.30944/20117582.574

Este es un artículo de acceso abierto bajo una Licencia Creative Commons - BY-NC-ND https://creativecommons.org/licenses/by-ncnd/4.0/deed.es 


\section{Introducción}

La vena porta es una de las principales venas del abdomen, y tiene por función drenar el flujo esplácnico al hígado, para el procesamiento de productos derivados de la absorción intestinal. Su oclusión genera cambios fisiológicos que producen un síndrome complejo llamado hipertensión portal, el cual puede tener complicaciones serias que amenazan la vida del paciente. Se debe clasificar muy bien al paciente con trombosis venosa portal ya que el tratamiento y el pronóstico son diferentes de acuerdo con el tiempo de evolución, enfermedades hepáticas asociadas y nivel anatómico.

La trombosis venosa portal extrahepática (TVPEH) es una entidad clínica en la que ocurre la trombosis de la vena porta extrahepática sin compromiso del parénquima hepático. Tiene diferentes causas, siendo las más comunes los síndromes mielodisplásicos y los trastornos de hipercoagulabilidad. En los niños, es muy frecuente el antecedente de canalización de la vena umbilical, que produce sepsis abdominal y onfalitis ${ }^{1}$. La primera descripción de esta entidad fue hecha por Elliot en 1895. Warren y Eberhard la caracterizaron en 1935 como una entidad clínica ${ }^{2}$. Los pacientes se presentan con hipertensión portal, asociada a varices esofágicas, ascitis y esplenomegalia.

Presentamos tres casos de TVPEH, con várices esofágicas severas, esplenomegalia, sangrado recurrente y dolor abdominal persistente, en quienes se practicó de manera exitosa derivación meso-Rex con vena yugular interna y vena femoral, con desaparición de los síntomas y ausencia de sangrado digestivo durante seis meses de seguimiento.

\section{Presentación de los casos Caso 1}

Paciente femenina de 14 años, procedente de zona rural del departamento de Arauca (Colombia), con antecedente de hemorragia de vías digestivas altas a repetición (3 - 5 por mes) desde los cuatro años de edad, con múltiples hospitalizaciones por anemia severa, con requerimiento de transfusión y ligadura endoscópica de varices esofágicas en varias oportunidades. Presentó nuevo episodio de sangrado con inestabilidad hemodinámica, por lo que fue remitida a nuestra institución. Informó ser producto de parto atendido en casa, con hospitalización neonatal por sepsis de origen abdominal y onfalitis, además de antecedente de trombosis venosa portal y degeneración cavernomatosa de la porta diagnosticada desde los 10 años. El desarrollo físico y sexual era normal para una mujer de su edad.

$\mathrm{Al}$ ingreso presentaba signos vitales normales, con palidez mucocutánea y esplenomegalia moderada no dolorosa; tenía hemoglobina de 7 gramos por decilitro $(\mathrm{g} / \mathrm{dL}$ ) y plaquetas de 65.500 por microlitro (mcL). Se realizó tomografía abdominal contrastada con fase portal, encontrando degeneración cavernomatosa de la porta (figura 1), y el doppler espleno-portal mostró vena porta de $8 \mathrm{~mm}$ de diámetro con evidencia de formaciones venosas colaterales a nivel del hilio hepático y esplenomegalia. Adicionalmente se realizó arteriografía de vasos abdominales y portografía retrograda que evidenciaron permeabilidad de la

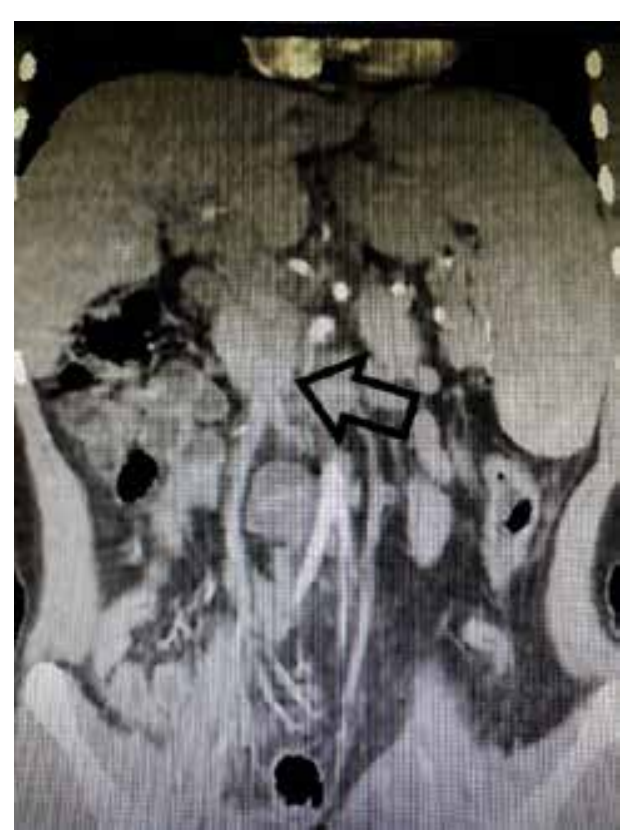

Figura 1. Corte coronal de tomografía de abdomen, donde se observa el cavernoma (flecha). 
arteria hepática, vena porta intrahepática y vena mesentérica superior. Se realizó biopsia hepática percutánea guiada por tomografía computarizada (TC), donde se observó normalidad del parénquima hepático. Debido a los hallazgos previos y al sangrado repetitivo, se indicó la realización de una derivación meso-Rex.

\section{Caso 2}

Paciente masculino de 16 años, residente en Bogotá, quien consultó por dolor abdominal súbito severo, sin antecedentes médicos o quirúrgicos previos, salvo consumo crónico de marihuana. Se llevó a laparotomía diagnostica por el servicio de cirugía general, encontrando varices congestivas en los compartimentos supra e infra meso cólico, con varices en todo el omento mayor y menor, pero sin sangrado activo (figura 2), e hígado de características normales. Debido a los hallazgos, no se realizó ningún procedimiento adicional.

Posteriormente, el paciente fue valorado por el servicio de cirugía vascular con sospecha de trombosis venosa portal, encontrando perfil hepático normal, endoscopia digestiva con várices esofágicas grado II, tomografía abdominal con

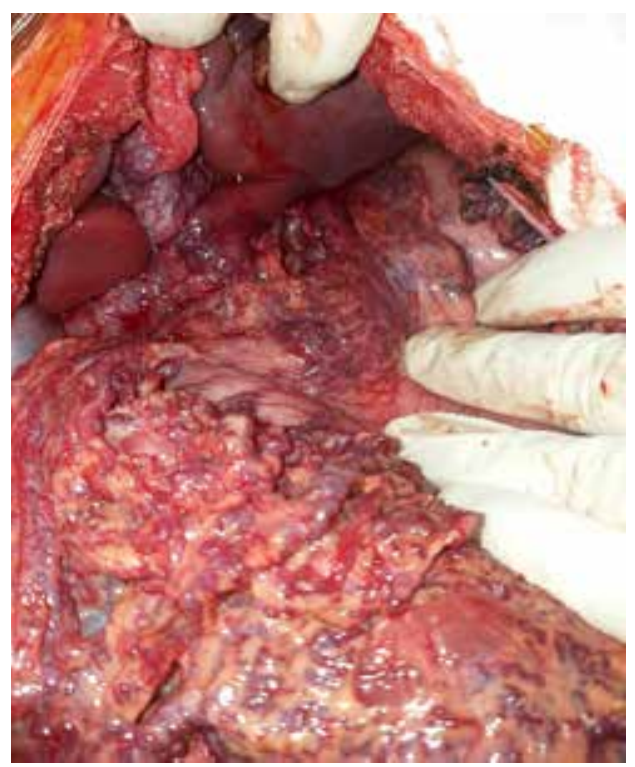

Figura 2. Várices en el epiplón mayor, menor y curvatura menor del estómago. un cavernoma portal, dúplex portal con degeneración cavernomatosa de la porta, y portografía retrograda normal. Se realizó una biopsia hepática percutánea guiada por TC que fue informada como normal.

Debido a la persistencia del dolor abdominal severo a pesar del uso de opiáceos, se decidió llevar al paciente a una derivación meso-Rex, cirugía sin complicaciones. Al tercer día posoperatorio, presentó dolor abdominal severo, descenso de la hemoglobina e hipotensión y la ecografía abdominal mostró liquido libre, por lo que se llevó a laparotomía, encontrando hemoperitoneo de $1000 \mathrm{ml}$ causado por sangrado de várices en el omento mayor, por lo que se realizó omentectomía parcial. El paciente evolucionó adecuadamente y se dio salida, con seguimiento normal hasta el sexto mes posoperatorio, y sin reaparición de los síntomas.

\section{Caso 3}

Paciente masculino de 14 años de edad, proveniente de zona rural del departamento de Tolima, con antecedente de degeneración cavernomatosa de la porta desde los 4 años, y múltiples episodios de sangrado digestivo, por lo que había requerido previamente dos procedimientos de ligadura de várices esofágicas sangrantes y biopsia hepática informada como normal. Además, retraso del crecimiento por debajo del percentil de peso y talla correspondiente a su edad. Consultó a nuestra institución por un nuevo episodio de sangrado digestivo alto, con dolor y distensión abdominal.

Al ingreso se documentó hiperesplenismo con trombocotipenia (17.000 plaquetas por $\mathrm{mcL}$ ) y esplenomegalia, se realizó una tomografía abdominal donde se observó una degeneración cavernomatosa de la porta y esplenomegalia masiva (figura 3). Por los episodios de sangrado frecuente y el hiperesplenismo, se decidió llevar a derivación meso-Rex, pero de forma preoperatoria bajo anestesia general se realizaron dos sesiones de embolización esplénica con coils y onyx, con el objetivo de mejorar el recuento de plaquetas y de disminuir el tamaño del bazo, para así lograr preservarlo (figuras $4 \mathrm{a}$ y $4 \mathrm{~b}$ ), logrando aumentar el recuento plaquetario a 192.500 por mcL. 
Posteriormente se realizó derivación meso-Rex sin complicaciones, pero a las 24 horas presentó distensión abdominal severa y anemia, por lo que requirió revisión quirúrgica encontrando hemoperitoneo de $600 \mathrm{cc}$ sin focos de sangrado activo y con adecuado estado de la reconstrucción vascular. El paciente evolucionó satisfactoriamente y se dio salida, sin presentar nuevos episodios de sangrado digestivo tras seis meses de seguimiento.

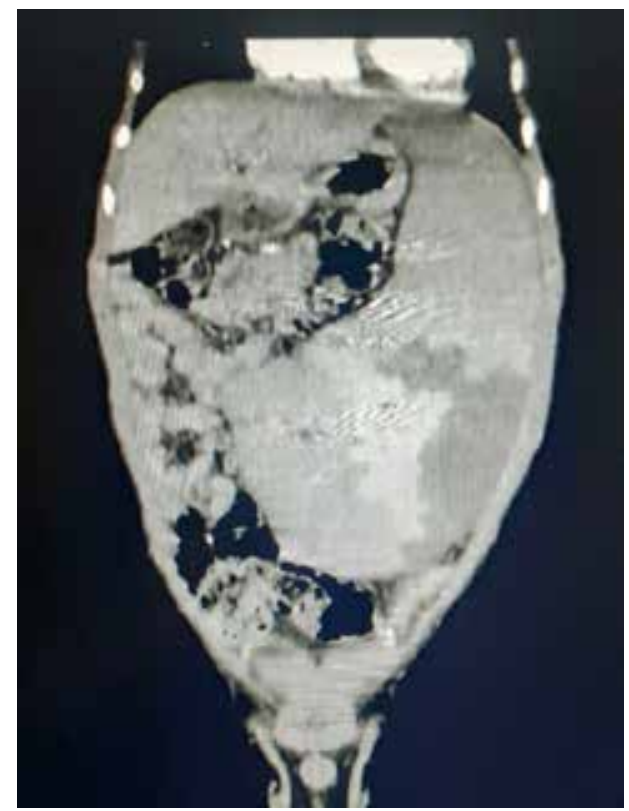

Figura 3. Esplenomegalia severa, que ocupa todo el hemi-abdomen izquierdo

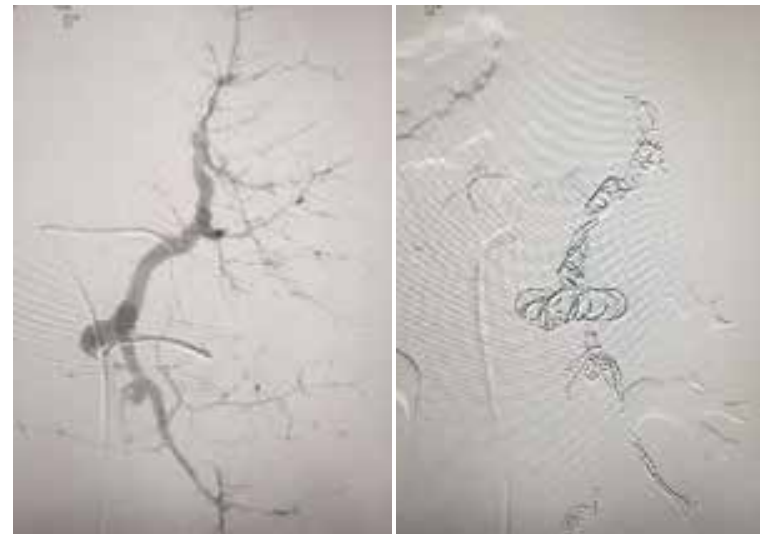

Figura 4a. Arteriografía de la arteria esplénica. 4b. Embolización y oclusión completa de la arteria esplénica con onyx y coils

\section{Técnica quirúrgica}

Se realizó bajo anestesia general, con monitoreo hemodinámico invasivo mediante línea arterial, catéter venoso central y sonda vesical. El abordaje a la cavidad abdominal se realizó por laparotomía subcostal bilateral (tipo Chevron), se procedió a la disección del ligamento redondo y de la placa hepática en la cisura de Rex con corte del parénquima hepático (figura 5), se disecó la vena porta izquierda, la cual era de buen diámetro y permeable en los tres casos. Se procedió a la disección de la vena mesentérica superior infra-mesocólica, de buen diámetro y permeable en los 3 pacientes.

Se tomó el injerto de vena yugular interna derecha con cervicotomía antero-lateral derecha amplia, obteniendo en los tres casos un segmento de vena largo pero insuficiente para realizar el injerto, por lo que fue necesario obtener un injerto de la vena femoral con la técnica de "Clagget", y construir un injerto uniendo estos dos segmentos venosos. Posteriormente se realizó anastomosis de la vena porta izquierda al injerto con puntos separados de Prolene ${ }^{\circledR}$ 6-0 (figura 6), se tunelizó el injerto por vía retro cólica y se realizó anastomosis con puntos separados de Prolene ${ }^{\circledR}$ 6-0 a la vena mesentérica superior, sin complicaciones (figura 7).

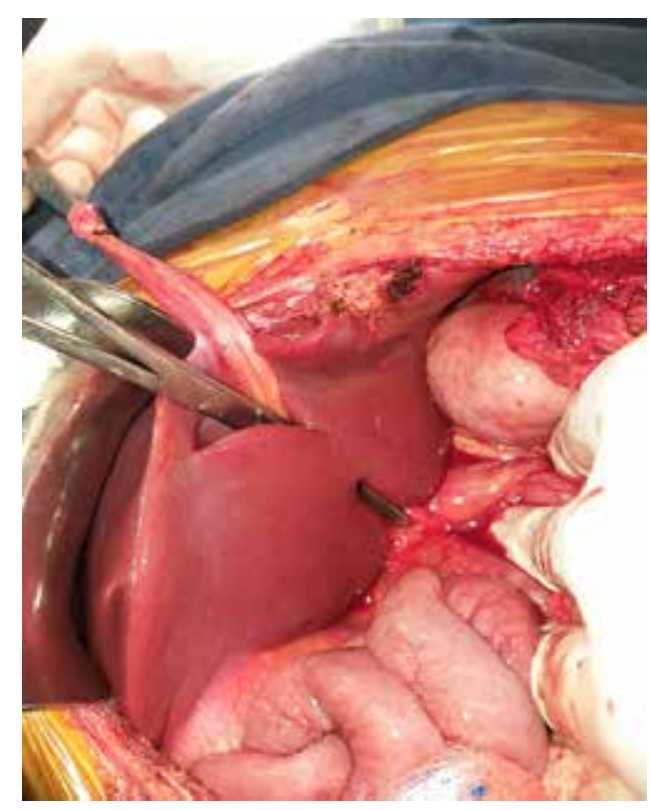

Figura 5. Cisura de Rex 


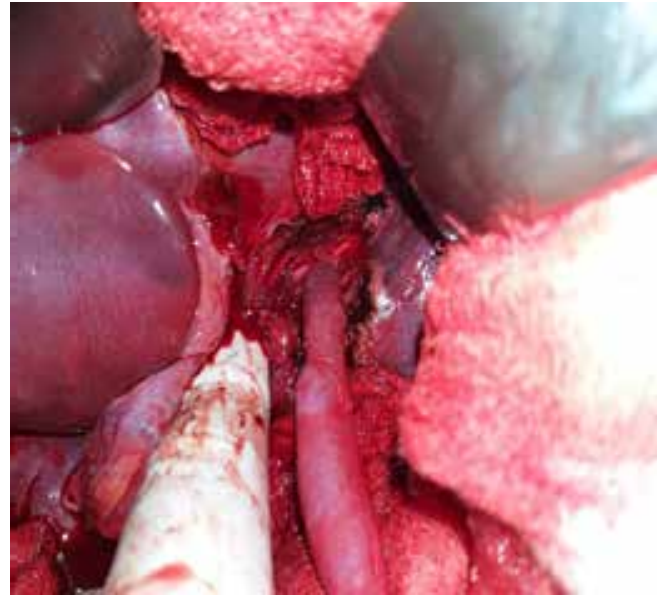

Figura 6. Anastomosis portal

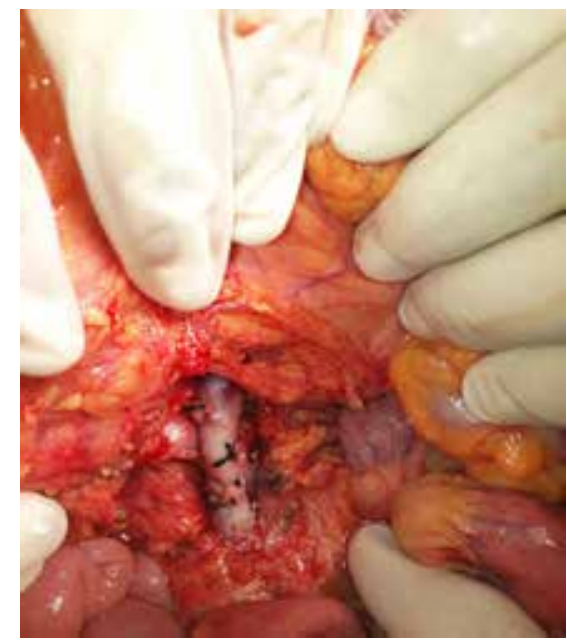

Figura 7. Anastomosis a la vena mesentérica superior.

El tiempo promedio de cirugía fue de tres horas, con un sangrado promedio de $400 \mathrm{ml}$. Todos los pacientes se trasladaron a la Unidad de Cuidados Intensivos en el posoperatorio, sin requerimiento de soporte ventilatorio ni inotrópico.

El seguimiento se hizo con hemogramas diarios, que mostraron aumento de las plaquetas y ausencia de anemia. La tomografía abdominal con fase portal y el doppler portal a los 10 días del procedimiento, mostró permeabilidad del injerto en los 3 casos (figura 8). La endoscopia digestiva a los 15 días mostró desaparición casi completa de las varices esofágicas, sin ningún signo de

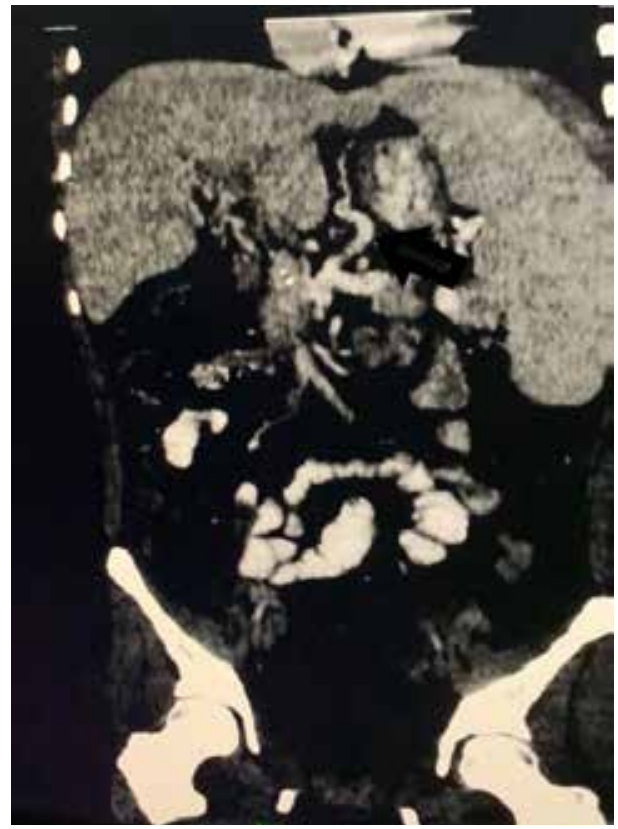

Figura 8. Angiotac de control donde se aprecia el injerto permeable.

sangrado. Durante los seis meses de seguimiento posteriores al tratamiento quirúrgico, ninguno de los tres pacientes volvió a presentar dolor abdominal, anemia o sangrado digestivo.

\section{Discusión}

Fisiológicamente, la oclusión venosa portal es bien tolerada. Al ocluirse esta vena, se pierde el $60 \%$ del flujo sanguíneo y el $20 \%$ del aporte de oxígeno al hígado, lo que genera vasodilatación arterial hepática y rescate venoso, que se refiere al desarrollo de circulación colateral para compensar la obstrucción. En la triada portal, estos vasos tienen la apariencia de un nudo irregular, simulando un cavernoma, que realmente no es la vena porta degenerada, sino que corresponde a las venas para-coledocianas del ligamento hepato-cólico y hepato-duodenal, las cuales se encuentran dilatadas en forma tortuosa alrededor de la vena porta. De allí el inadecuado, pero más conocido nombre de degeneración cavernomatosa de la porta ${ }^{3,4}$.

Esta circulación colateral se puede desarrollar entre tres y cinco semanas luego de ocurrida la 
obstrucción portal, y drenan la sangre de la circulación esplácnica a través de la vena gástrica izquierda, que también drena las venas de la unión gastroesofágica y del tercio distal del esófago, produciendo las várices esofágicas. La vena mesentérica inferior drena las venas hemorroidales, por lo que puede ocurrir la aparición de hemorroides internas ${ }^{3,4}$.

La trombosis venosa portal (TVP) es una entidad clínica compleja que genera hipertensión portal (gradiente de presión hepática venosa entre la vena cava y la vena porta mayor de $5 \mathrm{~mm} \mathrm{Hg}$ ). El riesgo de trombosis portal (TP) en la población genera es del $1 \%{ }^{5}$, y se puede presentar en diferentes escenarios clínicos, por lo que se debe clasificar de la siguiente manera, para determinar su tratamiento y pronóstico:

1. Tiempo de evolución: aguda (menor de seis meses) crónica (mayor de seis meses).

2. Asociada a cirrosis o malignidad intrahepática: la TP asociada a cirrosis ocurre en el 10 - $25 \%$ de los pacientes, y se caracteriza por la distorsión de la anatomía hepática, que disminuye el flujo venoso portal en volumen y velocidad. La asociada a neoplasias puede ser primaria del hígado, metastásica o por invasión a la porta. Los pacientes con hepatocarcinoma cursan con TP en un $44 \%$ de los casos y la mortalidad de la TP en estos casos es del $26 \%-30 \%{ }^{5}$.

3. No asociada a cirrosis o malignidad intrahepática: causada por síndromes mielodisplásicos (policitemia vera, trombocitemia esencial, mielofribrosis idiopática), trastornos de hipercoagulabilidad (mutación Janus Kinasa 2- JAK2, déficit de antitrombina III, niveles altos de homocisteína, déficit de proteína C y S, Factor de $\mathrm{V}$ de Leiden, uso de anticonceptivos orales), y por procesos inflamatorios locales adyacentes a la vena porta (pancreatitis, colecistitis, apendicitis, enfermedad inflamatoria intestinal, colangitis, sepsis abdominal por Bacteroides fragilis en niños con canalización de la vena umbilical y onfalitis). El $70 \%$ de los casos de
TP están asociados a síndromes de hipercoagulabilidad, por lo que se deben realizar siempre pruebas hematológicas. La mortalidad de la TP en estos casos es del $10 \%{ }^{6}$.

4. Anatómica: la vena porta, se forma por la confluencia de la vena mesentérica superior y la confluencia de la vena esplénica y de la vena mesentérica inferior, recibe a la vena gástrica izquierda, derecha, pancreático-duodenal supero-posterior, vena pre-pilórica y venas para umbilicales. Intra hepáticamente la vena porta derecha se divide en vena lateral derecha y en vena para mediana derecha, la vena porta izquierda se divide en vena lateral izquierda y en vena para mediana izquierda. La vena umbilical in útero lleva al hígado la sangre oxigenada de la placenta y al nacer se ocluye, convirtiéndose en el ligamento umbilical. Patológicamente la TP se divide en compromiso portal intrahepático, extrahepático o ambos ${ }^{7,8}$.

La Trombosis venosa portal extrahepática (TVPEH), es una forma crónica de TP, de origen vascular asociada a onfalitis. Es la principal causa de TP en niños con antecedente de partos atendidos en casa en países en vías de desarrollo, como en el caso número 1. Fue descrita en 1868 por Balfour y Stewart, y se caracteriza por la oclusión de la vena porta en el $75 \%$ de los casos, de las venas porta y esplénica en el $20 \%$, de las venas porta y mesentérica superior en el $5 \%$ y de todo el sistema porto-mesentérico y esplácnico en el 10\% de los casos. Entre más compromiso trombótico venoso exista de estas estructuras, mayor riesgo de isquemia y necrosis intestinal ${ }^{8,9,10}$.

No está asociada a cirrosis ni malignidad hepática y la función hepática es normal, tiene una mortalidad del $20 \%$ - $50 \%$, pero con el diagnóstico temprano y el uso de anticoagulación temprana y prolongada ha disminuido al $5 \%$, con una rata de sobrevida a 5 años del $85 \%$ y a 10 años del $81 \%$. La mortalidad está dada por la causa de obstrucción de la porta y no por las consecuencias de la hipertensión portal. En general, la trombosis aguda portal tiene un buen pronóstico en ausencia de infarto intestinal, que cuando ocurre, se asocia 
a falla multiorgánica y mortalidad en el $20 \%$ - 50

$\%$ de los casos. El sangrado digestivo se asocia con una baja mortalidad, en comparación con la que ocurren en los pacientes cirróticos, ya que en la obstrucción portal no cirrótica la función hepática esta conservada ${ }^{11,12}$.

\section{Manifestaciones clínicas}

La trombosis portal aguda se manifiesta por dolor abdominal, vómito, náuseas, fiebre, esplenomegalia y ascitis; la crónica por los mismos síntomas, pero, además por sangrado digestivo, presencia de varices en el esófago, duodeno, región anorrectal, lecho vesicular y ligamento hepato-duodenal, como consecuencia del desarrollo de la hipertensión portal ${ }^{13,14}$. En nuestros tres pacientes, el sangrado por las varices esofágicas fue el motivo de consulta.

La TVPEH se asocia a episodios recurrentes de sangrado digestivo alto, el cual es bien tolerado debido a que ocurre en pacientes jóvenes con función hepática normal. Se puede encontrar ascitis recurrente transitoria en el $25 \%$ de los niños, en el $10 \%$ de los casos puede haber esplenomegalia aislada, que puede ser sintomática, dada por dolor en el cuadrante superior izquierdo y $30 \%$ pueden tener hiperesplenismo (anemia y trombocitopenia). La encefalopatía no es común, debido a la presencia de derivaciones portosistémicos, aunque se puede presentar de manera transitoria después de un episodio de sangrado digestivo ${ }^{13,14}$. El tercer caso presentado se asoció a hiperesplenismo, razón por la cual se decidió realizar manejo endovascular con el objetivo de aumentar el recuento plaquetario. La esplenectomía en estos casos puede ser muy compleja y generalmente no se hace necesaria.

\section{Diagnostico}

Las pruebas de laboratorio no dan ninguna clave diagnostica en TP, por lo que los estudios imagenológicos son los más importantes:

Doppler portal: muestra turbulencia, inversión del flujo portal, presencia de material ecogénico dentro de la circulación portal, presencia de venas colaterales y esplenomegalia. La sensibilidad del
Doppler es del $80 \%$ - $100 \%$ y tiene una especificidad del $90 \%-100 \%{ }^{15,16}$.

Tomografía contrastada: se debe solicitar con fase portal para poder evaluar la circulación portal y la presencia del cavernoma, evidencia vasos tortuosos dilatados en la triada portal. Así mismo, permite evaluar estructuras vecinas y posible sufrimiento intestinal ${ }^{16,17}$.

Resonancia nuclear magnética: evalúa con mayor precisión las estructuras portales y el nivel de obstrucción, tiene una sensibilidad del $100 \%$ y una especificidad del $98 \%$ para diagnosticar TP. Igualmente se utiliza como examen de seguimiento para controlar intervenciones médicas o quirúrgicas en $\mathrm{TP}{ }^{18,19}$.

Portografía retrograda y Venografia transyugular: son procedimientos intervencionistas percutáneos con uso de angiografía, que permiten ver la permeabilidad de la circulación portal intra y extrahepática, pero con baja sensibilidad, dado que el medio de contraste se desvía por las venas dilatadas que derivan el flujo. Estos procedimientos se usan más como accesos para intervención endovascular, como trombólisis o trombectomía percutánea ${ }^{19-21}$. En nuestro caso se utilizó la portografía retrograda para valorar la permeabilidad de la vena porta izquierda intrahepática, como condición para poder realizar la derivación meso-Rex.

La biopsia hepática no se utiliza para diagnosticar trombosis portal, pero es importante para descartar patología hepática primaria o secundaria, especialmente antes de definir el tratamiento quirúrgico, ya que la derivación meso-Rex no es viable en casos de fibrosis hepática o de la vena porta intrahepática ${ }^{22,23}$. En nuestros pacientes, se contó con la biopsia, cuyo informe descartó patología parenquimatosa hepática.

\section{Tratamiento}

Los objetivos de tratamiento de los pacientes con TP, son:

1. Identificar la causa y definir posibles tratamientos definitivos. 
2. Tratar las complicaciones y las enfermedades concurrentes.

3. Prevenir trombosis futuras y crear estrategias que estimulen la recanalización vascular.

Aunque no hay estudios controlados, existe suficiente evidencia de que la anticoagulación temprana (iniciada a menos de una semana del inicio de los síntomas) es el tratamiento de entrada de la TP, logrando la recanalización en hasta el $49 \%$ de los pacientes, la cual puede ser tan baja como del $25 \%$ cuando se inicia de manera tardía ${ }^{22,23}$.

En las formas aguda y crónica, está indicada la anticoagulación plena por 1 año, y los estudios de hipercoagulabilidad son mandatorios. La trombólisis loco regional o indirecta a través de la arteria mesentérica superior, ha mostrado ser más efectiva y generar recanalizaciones más tempranas, sin embargo, aún no existen estudios aleatorizados y el riesgo de sangrado con los trombolíticos, pueden contraindicar su uso. Se recomienda, además, el uso de beta bloqueadores los cuales disminuyen el flujo y la presión esplácnica. El uso de anticoagulación plena no aumenta el riesgo de sangrado por varices esofágicas y la presencia de estas no contraindica el uso de la anticoagulación crónica 24-26.

La cirugía está indicada especialmente en los casos de sangrado digestivo recurrente, hiperesplenismo, isquemia y necrosis intestinal. Se emplean las derivaciones quirúrgicas, las cuales se dividen en selectivas y no selectivas ${ }^{26,27}$. Las selectivas (espleno-renal distal o de Warren-Zeppa y la meso-Rex), derivan el flujo portal al hígado, con baja probabilidad de encefalopatía. Las no selectivas (porto-cava termino lateral o latero lateral, espleno-renal proximal, meso-cava termino-lateral y los puentes de gran diámetro porto-cava o meso-cava), derivan el flujo portal fuera del hígado a la circulación sistémica, por lo que controlan el sangrado, pero generan encefalopatía. Finalmente existen los procedimientos no derivativos (devascularización gástrica, transección esofágica) que son poco recomendados ${ }^{26,27}$.

La derivación meso-Rex es una derivación fisiológica, ya que deriva el flujo mesentérico de manera directa a la circulación portal, sin paso de sangre a la circulación sistémica, lo que evita la encefalopatía. Se coloca un injerto autólogo de vena yugular interna o de vena femoral, entre la vena mesentérica superior y la vena porta izquierda intrahepática, que se ubica en el receso de Rex o cisura umbilical, el cual se ubica entre los segmentos hepáticos III y IV del lóbulo izquierdo. Esta vena generalmente es de buen diámetro y no se afecta por la circulación colateral. Fue diseñada por Ville de Goyet et al. en 1992, para obstrucción venosa portal luego de trasplante hepático, pero se ha popularizado en niños con trombosis venosa portal extrahepática. En adultos puede ser una buena opción, pero necesita aun validación científica ${ }^{28}$. La utilización de la vena yugular, ha mostrado tener la mejor permeabilidad para este tipo de derivación, pero su extracción está contraindicada en casos de malformaciones encefálicas tipo Arnold-Chiari o hidrocefalia ${ }^{29-31}$.

Las indicaciones del procedimiento son ${ }^{31-33}$ :

1. Sangrado recurrente por várices esofágicas, a pesar del tratamiento endoscópico.

2. Pacientes con sangrado por várices esofágicas que vivan en zonas rurales, apartadas de centros médicos de alta complejidad.

3. Esplenomegalia severa sintomática, con plaquetopenia menor a 10.000 por mcL.

4. Patología biliar portal.

5. Retardo severo del crecimiento.

Los requisitos indispensables para realizar esta derivación son: ausencia de enfermedades parenquimatosas hepáticas (especialmente cirrosis), diámetro de la vena porta izquierda mayor de $2 \mathrm{~mm}$ y permeabilidad de las venas mesentéricas ${ }^{34,35}$. En nuestros pacientes, la indicación del tratamiento quirúrgico estuvo dada por los episodios frecuentes de sangrado digestivo y por la no disponibilidad (por ser pacientes de áreas rurales) de centros cercanos de alta complejidad, para la atención en caso de emergencia. Las condiciones anatómicas fueron evaluadas por dúplex, portografía retrograda y tomografía con 
fase portal; igualmente se realizó biopsia percutánea previa al procedimiento, la cual determinó la normalidad del parénquima hepático.

Luego de la cirugía, los síntomas de hipertensión portal resuelven rápidamente, e incluso puede ocurrir que el tamaño del hígado aumente, y que el cavernoma disminuya de tamaño. El bazo puede disminuir o aumentar de tamaño, sin generar cambios hematológicos, pero en otros casos se puede presentar hiperesplenismo, por lo que se deben controlar diariamente las líneas celulares. El hiperesplenismo puede causar complicaciones hemorrágicas, caso en el que se debe realizar embolización del bazo por vía endovascular, la esplenectomía no es recomendada por el riesgo de sangrado y por las complicaciones infecciosas por gérmenes capsulados ${ }^{34,35}$. La esplenomegalia no es indicación de cirugía, pero se debe realizar esplenectomía durante el mismo acto quirúrgico, en caso de sangrado, de esplenomegalia masiva, o de esplenomegalia sintomática por dolor severo ${ }^{36}$.

La cirugía de meso-Rex modificado incluye variantes de la cirugía original, que se han realizado cuando la vena mesentérica superior no es permeable, obteniendo flujo de la vena gástrica izquierda, vena umbilical, vena mesentérica superior o vena esplénica. La vena esplénica o gástrica se puede anastomosar directamente rotándola hacia la vena porta izquierda sin requerir un injerto. En los casos en que no se encuentre la vena porta izquierda apta para realizar el puente, se debe optar como siguiente opción por una derivación espleno-renal distal con esplenectomía ${ }^{37-39}$.

\section{Seguimiento}

El seguimiento a largo plazo es muy importante, ya que son pacientes jóvenes que pueden presentar estenosis de la reconstrucción, generándose de nuevo hipertensión portal e hiperesplenismo. Se debe hacer seguimiento imagenológico mediante dúplex portal y si hay sospecha de estenosis se recomienda realizar angioresonancia y tomografía en fase portal. El tratamiento de la estenosis es percutáneo con angioplastias y si es necesario, mediante un nuevo puente ${ }^{39}$.

\section{Complicaciones de la TVPEH ${ }^{40-44}$ :}

Várices esofágicas: Las varices esofágicas de los pacientes con TP, se diferencian de las de los pacientes cirróticos, porque en la TP hay mucho menor riesgo de sangrado, pero pueden generar sangrados frecuentes o severos que amenazan la vida. La probabilidad de sangrado en TP no asociada a patología hepática es del $0,25 \%$ a dos años, mientras que si existe cirrosis es del $20 \%$ - $30 \%$ en el mismo periodo. El manejo endoscópico con bandas y ligadura controla el $90-95 \%$ de los episodios de sangrado, pero recurre en un $16 \%$, y tiene una mortalidad del $5 \%$. Las várices pueden tener una concurrencia en el estómago, duodeno, intestino delgado, región anorrectal, árbol biliar, sitios de anastomosis y estomas intestinales en hasta el $5 \%$ de los casos, y son de difícil manejo ${ }^{40}$.

Patología biliar portal: las várices que forman el cavernoma pueden comprometer la vía biliar, generando una biliopatía portal, que puede simular un colangiocarcinoma. En los estudios de colangiografía se pueden observar imágenes irregulares en la vía biliar, causadas por la compresión de los vasos dilatados, este es el llamado "signo del pseudo-colangiocarcinoma”, el cual es típico de la TP y se acompaña de ictericia y colestasis. El tratamiento es la decompresión del sistema portal por medio de derivaciones ${ }^{41}$.

Hiperesplenismo: ocurre cuando el bazo secuestra elementos formes de la sangre, causando anemia, leucopenia y plaquetopenia, es una consecuencia de la hipertensión portal y solucionar la misma, no garantiza que el hiperesplenismo ceda; el tamaño del bazo no está relacionado con la génesis del hiperesplenismo. Como complicación, pueden ocurrir hematomas espontáneos, cuando el recuento de plaquetas es inferior a $20.000 \mathrm{mcL}$, y sangrado interno con conteos menores a 5000 mcL. La leucopenia por sí sola no es tan grave, a menos que coexista con neutropenia. La principal indicación para tratar el hiperesplenismo es permitir el uso de medicamentos que supriman la medula ósea (interferón, agentes quimioterapéu- 
ticos). La mejor manera de disminuir la acción de secuestro del bazo es con embolización esplénica por vía endovascular (complicaciones menores al $5 \%$ ) y no con cirugía abierta (complicaciones del 10 - $30 \%$ ); la embolización tiene como objetivo generar necrosis del 50 - $70 \%$ del bazo. El hiperesplenismo puede mejorar hasta en el $50 \%$ de los casos, luego de la derivación portal ${ }^{42}$.

Retraso en el crecimiento: la anemia crónica puede generar retraso en el crecimiento en el $50 \%$ de los niños, debido a la alteración en la síntesis de factor de crecimiento IGF-1. Se ha sugerido también que el aumento desmesurado del bazo genera disminución del apetito por saciedad rápida y se asocia a desnutrición y retraso del crecimiento. Estos pacientes pueden presentar además disfunción cognitiva y motora moderada, y mala absorción debido a la enteropatía hipertensiva portal ${ }^{43}$.

Isquemia intestinal: ocurre en el $5 \%$ de casos de trombosis venosa portal y está asociada a trombosis extensas, que comprometen la vena mesentérica superior e inferior. Cuando hay signos de abdomen agudo o diarrea con sangre se debe intervenir prontamente al paciente para evitar peritonitis y falla multiorgánica; si se diagnostica al tiempo la obstrucción venosa y la necrosis intestinal, se puede realizar en el mismo tiempo quirúrgico la resección intestinal y la derivación portal ${ }^{44}$.

\section{Pronóstico}

En general, la trombosis aguda portal tiene un buen pronóstico, con una tasa de sobrevida a 5 años del $85 \%$. El pronóstico de la TP está dado por la causa de base que genere la trombosis y no por las complicaciones de la hipertensión portal. Se cree que el sangrado por várices esofágicas es una causa importante de mortalidad, pero gracias al manejo endoscópico, los nuevos estudios han mostrado resultados diferentes. El pronóstico de la TP en pacientes sin patología hepática es bueno, con tasas de sobrevida a 5 y 10 años del $90 \%$ y del $80 \%$ respectivamente. Cuando hay cirrosis, el pronóstico es peor, dado que la función hepática está comprometida. Cuando la etiología es neoplásica, el pronóstico es aún peor (sobrevida a 5 años del $8 \%$ ). Otro factor que tiene un impacto negativo en la sobrevida de la TP es la isquemia y la necrosis intestinal, que se relacionan con una mortalidad del $20 \%$ - $50 \%$. La presencia de un síndrome mielodisplásico no altera la sobrevida a 5 años ${ }^{45-47}$.

\section{Conclusión}

La trombosis de la vena porta es una enfermedad de origen multifactorial, de presentación muy variable y curso clínico relativamente benigno según la función hepática y la causa de base de la obstrucción. La TP aguda y crónica son entidades de diferente presentación y manejo. La anticoagulación es el principal pilar de manejo para la TP aguda y para la crónica, varía de acuerdo con las complicaciones asociadas por la hipertensión portal. La cirugía de meso-Rex es un procedimiento curativo para los casos de obstrucción extrahepática, documentado ampliamente en los niños, pero con probabilidades de aplicación en adultos. Se requieren más estudios para establecer los beneficios de las opciones terapéuticas disponibles.

\section{Cumplimiento de normas éticas}

Consentimiento informado: los autores confirman tener la autorización de los pacientes para su publicación.

Conflictos de interés: los autores declaran no tener ningún conflicto.

Fuente de Financiación: este trabajo fue autofinanciado por los autores.

Contribución de los autores: Diseño estudio: César Jiménez, Osiris Moreno.

Análisis: César Jiménez, Osiris Moreno, Mario Bravo.

Adquisición e interpretación de datos y redacción del manuscrito: César Jiménez, Leonardo Randial, Manuel Hossman, Iván Silva.

Todos los autores certifican que el manuscrito ha sido leído y aprobado. 


\section{Referencias}

1. Chawla Y, Bodh V. Portal Vein Thrombosis, Portal Vein Thrombosis. J Clin Exp Hepatol. 2015;5:22-40. https://doi.org/10.1016/j.jceh.2014.12.008

2. Boley SJ, Brant LJ, Sammartano RJ. History of mesenteric ischemia: evolution of a diagnosis and management. Surg Clin N Am. 1997;77:275-87. https://doi.org/10.1016/S0039-6109(05)70548-X

3. Webb LJ, Sherlock S. The aetiology, presentation and natural history of extra hepatic portal venous obstruction. Q J Med. 1979;192:627-39.

4. Ogren M, Bergqvist D, Bjorck M, Acosta S, Eriksson H, Sternby NH. Portal vein thrombosis: prevalence, patient characteristics and lifetime risk: a population study based on 23,796 consecutive autopsies. World J Gastroenterol. 2006;12:2115-9.

https://doi.org/10.3748/wjg.v12.i13.2115

5. Hoekstra H, Janssen L. Vascular liver disorders (II): portal vein thrombosis. J Med (Netherlands). 2009;67: 46-53.

6. Chawla Y, Duseja A, Dhiman K. Review article: the modern management of portal vein thrombosis. Aliment Pharmacol Ther. 2009;30:881-94. https://doi.org/10.1111/j.1365-2036.2009.04116.x

7. Yong-Pil C, Tae-Yong H, Gi-Young K, Kim K, Sung L. Use of meso-Rex shunt with transposition of the coronary vein for the management of extrahepatic portal vein obstruction. Ann Surg Treat Res. 2014;86:105-8. https://doi.org/10.4174/astr.2014.86.2.105

8. Franchis R, Baveno VI Faculty. Expanding consensus in portal hypertension: Report of the Baveno VI Consensus Workshop: Stratifying risk and individualizing care for portal hypertension. J Hepatol. 2015;63:743-52. https://doi.org/10.1016/j.jhep.2015.05.022

9. Franchis R, Pascal JP, Ancona E, Burroughs AK, Henderson JM, Fleig W, et al. Definitions, methodology and therapeutic strategies in portal hypertension. A consensus development workshop, Baveno, Lake Maggiore, Italy, April 5 and 6, 1990. J Hepatol. 1992;15:256-61. https://doi.org/10.1016/0168-8278(92)90044-P

10. Garcia-Tsao G, Bosch J, Groszmann R. Portal hypertension and variceal bleeding, unresolved issues. Summary of an American Association for the study of liver disease and of the European Association for the Study of the Liver single-topic conference. Hepatology. 2008;47:176472. https://doi.org/10.1002/hep.22273

11. Seijo-Rios S, Garcia-Pagan JC. Trombosis portal. Gastroenterol Hepatol. 2010;33:179-90. https://doi.org/10.1016/j.gastrohep.2009.04.002

12. Webster G, Burroughs K, Riordan M. Review article: portal vein thrombosis - new insights into aetiology and management. Aliment Pharmacol Ther. 2005;21:1-9. https://doi.org/10.1111/j.1365-2036.2004.02301.x
13. Wolter K, Decker G, Kuetting D, Trebicka J, Manekeller S. Interventional treatment of acute portal vein thrombosis. Fortschr Röntgenstr. 2018;190:740-6. https://doi.org/10.1055/a-0631-9265

14. Lang S, Loss M, Wohlgemuth W, Schlift H. Clinical management of acute portal/mesenteric vein thrombosis. Viszeralmedizin. 2014;30:394-400. https://doi.org/10.1159/000369896

15. Claudon M, Dietrich CF, Choi BI, Cosgrove DO, Kudo M, Nolsøe CP, et al. Guidelines and good clinical practice recommendations for contrast enhanced ultrasound (CEUS) in the liver - update 2012: a WFUMB-EFSUMB initiative in cooperation with representatives of AFSUMB, AIUM, ASUM, FLAUS and ICUS. Ultraschall Med. 2013; 34:11-29. https://doi.org/10.1055/s-0032-1325499

16. Parenti C. Evaluation of Rex Shunts: What Sonographers Need to Know J Diagnostic Medical Sonography. 2013;29:238-42. https://doi.org/10.1177/8756479313497429

17. Berzigotti A, Garcia-Criado A, Darnell A, Garcia- Pagan JC. Imaging in clinical decision-making for portal vein thrombosis. Nat Rev Gastroenterol Hepatol. 2014;11:308-16. https://doi.org/10.1038/nrgastro.2013.258

18. Singal AK, Kamath PS, Tefferi A. Mesenteric venous thrombosis. Mayo Clin Proc. 2013;88:285-94. https://doi.org/10.1016/j.mayocp.2013.01.012

19. Tae-Yong H, Kim H, Gi-Young K, Seak O. Variant meso-Rex bypass with transposition of abdominal autogenous vein for the management of idiopathic extrahepatic portal vein obstruction: a retrospective observational study. BMC Surgery. 2015;15:116. https://doi.org/10.1186/s12893-015-0101-6

20. Sundeep J, Kalla M, Suleman A. Proximal spleno-renal shunt with retroaortic left renal vein in a patient with extrahepatic portal vein obstruction: first case report. BMC Surgery. 2017;17:65. https://doi.org/10.1186/s12893-017-0262-6

21. Wang M, Lin H, Guo L, Liu F. Acute extensive portal and mesenteric venous thrombosis after splenectomy: Treated by interventional thrombolysis with transjugular approach, World J Gastroenterol. 2009;15:3038-45. https://doi.org/10.3748/wjg.15.3038

22. Turnes J, Garcia-Pagan JC, Gonzalez M, Aracil C, Calleja JL, Ripoll C, et al. Portal hypertension-related complications after acute portal vein thrombosis: Impact of early anticoagulation. Clin Gastroenterol Hepatol. 2008;6:1412-7. https://doi.org/10.1016/j.cgh.2008.07.031

23. Plessier A, Murad SD, Hernandez-Guerra M, Consigny Y, Fabris F, Heller J, et al. A prospective multicentric follow up study on 105 patients with acute portal vein throm- 
bosis: results from EN-VIE. Hepatology. 2007;46(suppl):310A.

24. Chung JW, Kim GH, Lee JH, Ok KS, Jang ES, Jeong SH, et al. Safety, efficacy, and response predictors of anticoagulation for the treatment of nonmalignant portal-vein thrombosis in patients with cirrhosis: a propensity score matching analysis. Clin Mol Hepatol. 2014;20:38491. https://doi.org/10.3350/cmh.2014.20.4.384

25. Rosenquist K, Eriksson LG, Rorsman F, Sangfelt P, Nyman R. Endovascular treatment of acute and chronic portal vein thrombosis in patients with cirrhotic and non-cirrhotic liver. Acta Radiol. 2016;57:572-9. https://doi.org/10.1177/0284185115595060

26. Kim HS, Patra A, Khan J, Arepally A, Streiff MB. Transhepatic catheter-directed thrombectomy and thrombolysis of acute superior mesenteric venous thrombosis. J Vasc Interv Radiol. 2005;16:1685-91. https://doi.org/10.1097/01.RVI.0000182156.71059.B7

27. Harmanci O, Bayraktar Y. Portal hypertension due to portal venous thrombosis: Etiology, clinical outcomes. World J Gastroenterol. 2007;13: 2535-40. https://doi.org/10.3748/wjg.v13.i18.2535

28. de Goyet J, Clapuyt P, Otte JB. Extrahilar mesenterico-left portal shunt to relieve extrahepatic portal hypertension after partial liver transplant. Transplantation. 1992;53:231-2.

29. Chiu B, Pillai SB, Sandler AD, Superina RA. Experience with alternate sources of ve $\neg$ nous inflow in the meso-Rex bypass operation: the coronary and splenic veins. J Pediatr Surg. 2007;42:1199-202. https://doi.org/10.1016/j.jpedsurg.2007.02.033

30. Prasad A, Gupta S, Kohli V, Girish K. Proximal Splenorenal Shunts for Extrahepatic Portal Venous Obstruction in Children. Ann Surg. 1994;219:193-6. https://doi.org/10.1097/00000658-199402000-00011

31. López-Santamaría M, Gámez M, Murcia J, Frauca E, Hierro L, Camarena C, de la Vega A, et al. Hipertensión portal prehepática como complicación tardía del trasplante hepático pediátrico Cir Pediatr 2001;14:135-8.

32. Caronna R, Bezzi M, Schiratti M, Cardi M, Prezioso G, Benedetti M, et al. Severe bleeding from esophageal varices resistant to endoscopic treatment in a non cirrhotic patient with portal hypertension. World J Emerg Surg. 2008,3:24.

https://doi.org/10.1186/1749-7922-3-24

33. Hau M, Fellmer P, Schoenberg M, Schmelzle M, Morgul $\mathrm{M}$. The collateral caval shunt as an alternative to classical shunt procedures in patients with recurrent duodenal varices and extrahepatic portal vein thrombosis. Eur J Med Res. 2014;19:36. https://doi.org/10.1186/2047-783X-19-36

34. Belli L, Puttini M, Marni A: Extrahepatic portal obstruction: clinical experience and surgical treatment in 105 patients. J Cardiovasc Surg (Torino). 1980;21:439-48.

35. Han D, Tang R, Wang L, Li A, Huang X, Shen S, et al. Case report of a modified Meso-Rex bypass as a treatment technique for late-onset portal vein cavernous transformation with portal hypertension after adult deceased-donor liver transplantation. Medicine. 2017;96:e7208. https://doi.org/10.1097/MD.0000000000007208

36. Sobhonslidsuk A, Reddy KR. Portal vein thrombosis: a concise review. Am J Gastroenterol. 2002;97:535-41. https://doi.org/10.1111/j.1572-0241.2002.05527.x

37. Wei Z, Guang R, Zhang Y, Li D, Liu Q. Partial splenectomy and use of splenic vein as an autograft for meso-Rex bypass: A clinical observational study. Med Sci Monit. 2014;20:2235-42.

https://doi.org/10.12659/MSM.892482

38. Superina R, Bambini DA, Lokar J, Rigsby C, Whitington PF. Correction of extrahepatic portal vein thrombosis by the mesenteric to left portal vein bypass. Ann Surg. 2006;243:515-21.

https://doi.org/10.1097/01.sla.0000205827.73706.97

39. Domínguez-Amillo C, De la Torre R, Moreno A, Encinas-Hernandez JL. Resultados del shunt mesoportal de Rex en el tratamiento de la trombosis portal extrahepática idiopática. Cir Pediatr. 2017;30:22-7.

40. Salzedas-Netto AA, Duarte AA, Linhares MM, Mattar RH, Medeiros KL, Cury EK, et al. Variation of the Rex shunt for treating concurrent obstruction of the portal and superior mesenteric veins. J Pediatr Surg. 2011;46:201820. https://doi.org/10.1016/j.jpedsurg.2011.07.002

41. Ateş O, Hakgüder G, Olguner M, Seçil M, Karaca I, Akgür FM. Mesenterico left portal bypass for variceal bleeding owing to extrahepatic portal hypertension caused by portal vein thrombosis. J Pediatr Surg. 2006;41:1259-63 https://doi.org/10.1016/j.jpedsurg.2006.03.043

42. Zhang H, Zhang N, Li M, Jin W, Pan S. Surgical treatment of portal vein cavernous transformation. World J Surg. 2004;28:708-11. https://doi.org/10.1007/s00268-004-7265-z

43. Boyer TD, Habib S. Big spleens and hypersplenism: fix it or forget it? Liver International. 2015;35:1492-8. https://doi.org/10.1111/liv.12702

44. Omer S, Zara O, Lacobescu C, Dina I. Partial splenic embolization for hypersplenism in cirrhotic patients. A case series. J Gastrointestin Liver Dis. 2014;23:215-8. https://doi.org/10.15403/jgld.2014.1121.232.so1

45. Kumar S, Sarr MG, Kamath PS. Mesenteric venous thrombosis. N Engl J Med. 2001;345:1683-8. https://doi.org/10.1056/NEJMra010076

46. Poddar U, Thapa BR, Singh K. Band ligation plus sclerotherapy versussclerotherapy alone in children with extrahepatic portal venous obstruction. J Clin Gastroenterol. 2005;39:626-9.

https://doi.org/10.1097/01.mcg.0000170765.36825.66

47. Spaander MC, Darwish Murad S, van Buuren HR, Hansen BE, Kuipers EJ, Janssen HL. Endoscopic treatment of esophagogastric variceal bleeding in patients with noncirrhotic extrahepatic portal vein thrombosis: a long-term follow-up study. Gastrointest Endosc. 2008;67:821-7.

https://doi.org/10.1016/j.gie.2007.08.023 